\title{
Binding of gold nanoclusters with size-expanded DNA bases: A computational study of structural and electronic properties.
}

\section{Purshotam Sharma, Himanshu Singh, Sitansh Sharma and Harjinder Singh*}

Center for computational natural Sciences and bioinformatics, International Institute of Information and Technology, Gachibowli, Hyderabad-500032, India

\section{Supporting Information}

Table S1. Optimized Coordinates of Gold-Complexed x-bases

\begin{tabular}{crrr}
$\mathrm{XA}-\mathrm{Au}_{6}$ & & \\
\hline $\mathrm{C}$ & -1.30415800 & -0.47716600 & -0.00048800 \\
$\mathrm{C}$ & -1.06389600 & 0.92659600 & -0.00065800 \\
$\mathrm{C}$ & 0.28947000 & 1.46010300 & -0.00085300 \\
$\mathrm{C}$ & 1.40827700 & 0.58523800 & -0.00081300 \\
$\mathrm{C}$ & 1.16023200 & -0.79142100 & -0.00056400 \\
$\mathrm{C}$ & -0.17949500 & -1.31144100 & -0.00042900 \\
$\mathrm{~N}$ & -2.17483400 & 1.83550700 & -0.00070800 \\
$\mathrm{C}$ & -1.88435300 & 3.19179700 & -0.00084800 \\
$\mathrm{~N}$ & -0.65774000 & 3.81590100 & -0.00103200 \\
$\mathrm{C}$ & 0.43092300 & 2.92853900 & -0.00110000 \\
$\mathrm{~N}$ & 2.09352700 & -1.89438000 & -0.00036300 \\
$\mathrm{C}$ & 1.33317600 & -3.02747900 & -0.00022600 \\
$\mathrm{~N}$ & -0.03236000 & -2.72775700 & -0.00036700 \\
$\mathrm{~N}$ & 1.66264300 & 3.53769700 & -0.00157000 \\
$\mathrm{H}$ & 1.72938600 & -4.05612200 & -0.00005800 \\
$\mathrm{H}$ & 1.68810800 & 4.57939900 & -0.00147000 \\
$\mathrm{H}$ & 2.57143300 & 3.01968100 & -0.00124100 \\
$\mathrm{H}$ & -2.76825400 & 3.85751000 & -0.00083300 \\
$\mathrm{H}$ & 2.44726200 & 0.95489700 & -0.00100600 \\
$\mathrm{H}$ & -2.33282100 & -0.87258100 & -0.00060000 \\
$\mathrm{H}$ & -0.80766600 & -3.43026500 & -0.00039400 \\
$\mathrm{Au}$ & -4.17684600 & 1.19967900 & -0.00023000 \\
$\mathrm{Au}$ & -6.80232800 & 0.43662700 & 0.00177600 \\
\hline & -5.21176100 & -1.98836000 & -0.00127000 \\
$\mathrm{Au}$ & 4.17873500 & -1.57334300 & 0.00004400 \\
\hline & 5.16983300 & 1.88187300 & -0.00044100 \\
& & & \\
$\mathrm{Au}$ & &
\end{tabular}

\begin{tabular}{crrr}
$\mathrm{xC}-\mathrm{Au}_{6}$ & & & \\
\hline $\mathrm{C}$ & 2.04370300 & 5.31131100 & -0.00003200 \\
$\mathrm{C}$ & 0.73150900 & 4.81676900 & 0.00048800 \\
$\mathrm{C}$ & 0.47422000 & 3.41257500 & 0.00066400 \\
$\mathrm{C}$ & 1.58331000 & 2.50963100 & 0.00014400 \\
$\mathrm{C}$ & 2.91924800 & 2.99886000 & -0.00048300 \\
$\mathrm{C}$ & 3.13194900 & 4.37980500 & -0.00055200 \\
$\mathrm{C}$ & -0.88523100 & 2.82389600 & 0.00142000 \\
$\mathrm{~N}$ & -1.06622100 & 1.45052700 & 0.00165900 \\
$\mathrm{C}$ & 0.04730600 & 0.56991600 & 0.00100400 \\
$\mathrm{~N}$ & 1.33726100 & 1.10272800 & 0.00059700 \\
$\mathrm{O}$ & -0.28329200 & -0.72506600 & 0.00078200 \\
$\mathrm{~N}$ & -2.02485400 & 3.58194600 & 0.00228200 \\
$\mathrm{C}$ & 2.32747300 & 6.82171300 & 0.00048700 \\
$\mathrm{H}$ & -2.95444900 & 3.10281400 & 0.00232200 \\
$\mathrm{H}$ & -1.99089000 & 4.62004700 & 0.00191600 \\
$\mathrm{H}$ & 2.15219400 & 0.43026100 & 0.00052600 \\
$\mathrm{H}$ & -0.10659300 & 5.52661500 & 0.00073600 \\
$\mathrm{H}$ & 4.16459000 & 4.76050300 & -0.00112200 \\
$\mathrm{H}$ & 2.90771800 & 7.10202600 & 0.89577400 \\
$\mathrm{H}$ & 2.92051800 & 7.10037300 & -0.88680300 \\
$\mathrm{H}$ & 1.38973600 & 7.39602100 & -0.00660600 \\
$\mathrm{H}$ & 3.76216400 & 2.28697600 & -0.00131100 \\
$\mathrm{Au}$ & -2.73897800 & 0.00040000 & 0.09950800 \\
$\mathrm{Au}$ & -4.76483700 & -1.74146900 & 0.00031500 \\
$\mathrm{Au}$ & -5.52786400 & 1.12008300 & -0.00107900 \\
$\mathrm{Au}$ & 4.19841400 & -1.25834600 & 0.00040000 \\
$\mathrm{Au}$ & 6.35014900 & 0.38744500 & -0.00060200 \\
$\mathrm{Au}$ & 1.57244700 & -2.16846500 & -0.00022300 \\
\hline & & & \\
\hline & & &
\end{tabular}




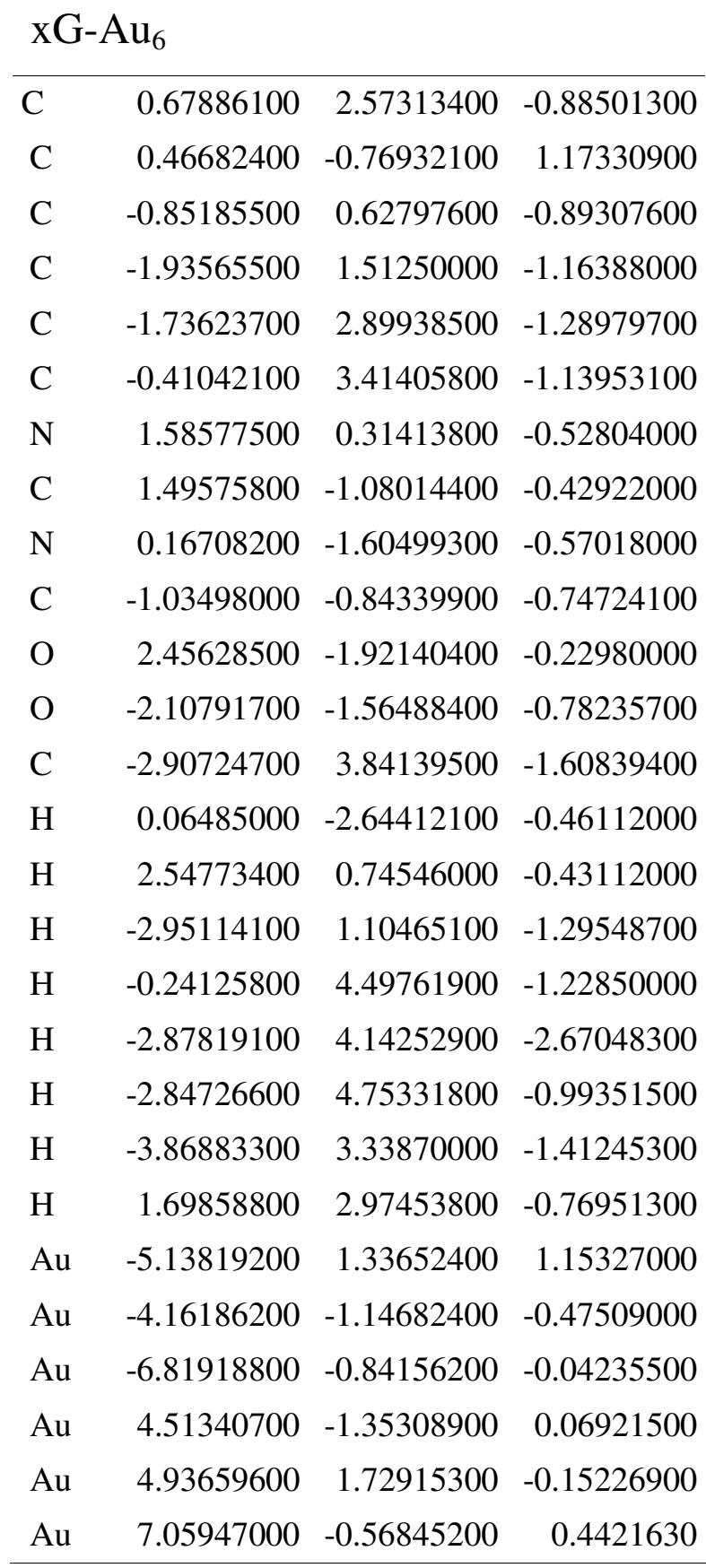

\begin{tabular}{crrr}
$\mathrm{XT}-\mathrm{Au}_{6}$ & & & \\
\hline $\mathrm{C}$ & 0.67886100 & 2.57313400 & -0.88501300 \\
$\mathrm{C}$ & 0.46682400 & 1.17330900 & -0.76932100 \\
$\mathrm{C}$ & -0.85185500 & 0.62797600 & -0.89307600 \\
$\mathrm{C}$ & -1.93565500 & 1.51250000 & -1.16388000 \\
$\mathrm{C}$ & -1.73623700 & 2.89938500 & -1.28979700 \\
$\mathrm{C}$ & -0.41042100 & 3.41405800 & -1.13953100 \\
$\mathrm{~N}$ & 1.58577500 & 0.31413800 & -0.52804000 \\
$\mathrm{C}$ & 1.49575800 & -1.08014400 & -0.42922000 \\
$\mathrm{~N}$ & 0.16708200 & -1.60499300 & -0.57018000 \\
$\mathrm{C}$ & -1.03498000 & -0.84339900 & -0.74724100 \\
$\mathrm{O}$ & 2.45628500 & -1.92140400 & -0.22980000 \\
$\mathrm{O}$ & -2.10791700 & -1.56488400 & -0.78235700 \\
$\mathrm{C}$ & -2.90724700 & 3.84139500 & -1.60839400 \\
$\mathrm{H}$ & 0.06485000 & -2.64412100 & -0.46112000 \\
$\mathrm{H}$ & 2.54773400 & 0.74546000 & -0.43112000 \\
$\mathrm{H}$ & -2.95114100 & 1.10465100 & -1.29548700 \\
$\mathrm{H}$ & -0.24125800 & 4.49761900 & -1.22850000 \\
$\mathrm{H}$ & -2.87819100 & 4.14252900 & -2.67048300 \\
$\mathrm{H}$ & -2.84726600 & 4.75331800 & -0.99351500 \\
$\mathrm{H}$ & -3.86883300 & 3.33870000 & -1.41245300 \\
$\mathrm{H}$ & 1.69858800 & 2.97453800 & -0.76951300 \\
$\mathrm{Au}$ & -5.13819200 & 1.33652400 & 1.15327000 \\
$\mathrm{Au}$ & -4.16186200 & -1.14682400 & -0.47509000 \\
$\mathrm{Au}$ & -6.81918800 & -0.84156200 & -0.04235500 \\
$\mathrm{Au}$ & 4.51340700 & -1.35308900 & 0.06921500 \\
\hline & 4.93659600 & 1.72915300 & -0.15226900 \\
$\mathrm{Au}$ & 7.05947000 & -0.56845200 & 0.44216300 \\
\hline & & & \\
$\mathrm{H}$ & &
\end{tabular}


TABLE S2: Bondlengths before and after complex formation with gold atoms

\begin{tabular}{|c|c|c|c|c|c|c|c|c|c|c|c|}
\hline Bond. & $x A$ & $x A-A u_{6}$ & Bond & $x C$ & $x C-A u_{6}$ & Bond & $x G$ & $x G-A u_{6}$ & Bond & $x T$ & $x T-A u_{6}$ \\
\hline $\mathrm{C} 1-\mathrm{H} 20$ & 1.07 & 1.10 & C1-C13 & 1.51 & 1.54 & $\mathrm{C} 1-\mathrm{H} 21$ & 1.07 & 1.10 & C1-H21 & 1.08 & 1.10 \\
\hline C1-C6 & 1.37 & 1.40 & $\mathrm{C} 1-\mathrm{C} 2$ & 1.37 & 1.40 & $\mathrm{C} 1-\mathrm{C} 2$ & 1.39 & 1.41 & $\mathrm{C} 1-\mathrm{C} 2$ & 1.39 & 1.42 \\
\hline $\mathrm{C} 1-\mathrm{C} 2$ & 1.40 & 1.42 & C1-C6 & 1.40 & 1.43 & C1-C6 & 1.38 & 1.40 & $\mathrm{C} 2-\mathrm{C} 3$ & 1.39 & 1.43 \\
\hline C6-C5 & 1.41 & 1.44 & C2-H17 & 1.07 & 1.10 & C5-C6 & 1.41 & 1.44 & $\mathrm{C} 3-\mathrm{C} 4$ & 1.39 & 1.42 \\
\hline $\mathrm{C} 5-\mathrm{C} 4$ & 1.37 & 1.40 & C2-C3 & 1.40 & 1.43 & C4-C5 & 1.37 & 1.41 & C4-H16 & 1.07 & 1.10 \\
\hline C4-H19 & 1.07 & 1.10 & C3-C4 & 1.39 & 1.43 & $\mathrm{C} 4-\mathrm{H} 20$ & 1.07 & 1.10 & C4-C5 & 1.38 & 1.41 \\
\hline $\mathrm{C} 4-\mathrm{C} 3$ & 1.40 & 1.42 & C4-C5 & 1.40 & 1.42 & $\mathrm{C} 4-\mathrm{C} 3$ & 1.39 & 1.42 & C5-C13 & 1.51 & 1.54 \\
\hline $\mathrm{C} 3-\mathrm{C} 2$ & 1.41 & 1.45 & $\mathrm{C} 5-\mathrm{H} 22$ & 1.08 & 1.10 & $\mathrm{C} 2-\mathrm{C} 3$ & 1.42 & 1.45 & C13-H18 & 1.09 & 1.10 \\
\hline N13-C6 & 1.37 & 1.42 & C5-C6 & 1.37 & 1.40 & C9-N10 & 1.28 & 1.37 & C13-H19 & 1.09 & 1.10 \\
\hline N13-H21 & 0.99 & 1.04 & C6-H18 & 1.08 & 1.10 & C2-N10 & 1.38 & 1.45 & C13-H20 & 1.08 & 1.10 \\
\hline N13-C12 & 1.37 & 1.40 & C3-C7 & 1.47 & 1.48 & $\mathrm{C} 3-\mathrm{C} 7$ & 1.46 & 1.48 & C5-C6 & 1.40 & 1.43 \\
\hline C12-H15 & 1.07 & 1.10 & C7-N12 & 1.32 & 1.37 & C7-O15 & 1.21 & 1.30 & C6-H17 & 1.08 & 1.10 \\
\hline C12-N11 & 1.28 & 1.36 & C7-N8 & 1.31 & 1.39 & C7-N8 & 1.37 & 1.43 & C6-C1 & 1.38 & 1.40 \\
\hline N11-C5 & 1.39 & 1.44 & N8-C9 & 1.36 & 1.42 & N8-C9 & 1.37 & 1.42 & C2-N7 & 1.38 & 1.43 \\
\hline $\mathrm{N} 7-\mathrm{C} 2$ & 1.37 & 1.44 & C9-O11 & 1.21 & 1.34 & N8-H19 & 1.01 & 1.05 & N17-H15 & 0.99 & 1.06 \\
\hline N7-C8 & 1.28 & 1.39 & C9-N10 & 1.37 & 1.40 & C9-N14 & 1.34 & 1.40 & N7-C8 & 1.37 & 1.40 \\
\hline $\mathrm{C} 3-\mathrm{C} 10$ & 1.45 & 1.48 & N10-H16 & 0.99 & 1.06 & N14-H18 & 0.99 & 1.05 & C8-O11 & 1.20 & 1.29 \\
\hline C10 -N14 & 1.34 & 1.37 & N10-C4 & 1.37 & 1.43 & N14-H17 & 1.00 & 1.05 & C8-N9 & 1.37 & 1.43 \\
\hline C10-N9 & 1.31 & 1.40 & N12-H15 & 0.99 & 1.04 & N13-C12 & 1.37 & 1.42 & N9-H14 & 1.01 & 1.05 \\
\hline N9-C8 & 1.35 & 1.38 & N12-H14 & 1.01 & 1.05 & N13-C6 & 1.37 & 1.41 & N9-C10 & 1.37 & 1.43 \\
\hline C8-H18 & 1.08 & 1.11 & C13-H19 & 1.08 & 1.10 & N13-H22 & 0.99 & 1.04 & C10-O12 & 1.20 & 1.29 \\
\hline N14-H16 & 1.00 & 1.04 & $\mathrm{C} 13-\mathrm{H} 20$ & 1.09 & 1.10 & C12-H16 & 1.07 & 1.10 & $\mathrm{C} 10-\mathrm{C} 3$ & 1.47 & 1.49 \\
\hline N14-H17 & 0.99 & 1.04 & $\mathrm{C} 13-\mathrm{H} 21$ & 1.08 & 1.10 & C12-N11 & 1.27 & 1.35 & $\mathrm{Au} 22-\mathrm{Au} 23$ & & 3.13 \\
\hline N7-Au22 & & 2.10 & N8-Au23 & & 2.15 & C5-N11 & 1.39 & 1.45 & $\mathrm{Au} 23-\mathrm{Au} 24$ & & 2.71 \\
\hline $\mathrm{Au} 22-\mathrm{Au} 24$ & & 3.35 & $\mathrm{Au} 23-\mathrm{Au} 24$ & & 2.74 & $\mathrm{Au} 25-\mathrm{Au} 28$ & & 2.67 & $\mathrm{Au} 24-\mathrm{Au} 22$ & & 2.30 \\
\hline $\mathrm{Au} 22-\mathrm{Au} 23$ & & 2.73 & $\mathrm{Au} 24-\mathrm{Au} 25$ & & 2.96 & $\mathrm{Au} 23-\mathrm{Au} 24$ & & 2.67 & $\mathrm{O} 12-\mathrm{Au} 23$ & & 2.12 \\
\hline $\mathrm{Au} 23-\mathrm{Au} 24$ & & 2.90 & $\mathrm{Au} 23-\mathrm{Au} 25$ & & 2.97 & $\mathrm{Au} 26-\mathrm{Au} 27$ & & 2.68 & $\mathrm{Au} 25-\mathrm{Au} 26$ & & 3.12 \\
\hline N11-Au25 & & 2.11 & $\mathrm{Au} 28-\mathrm{Au} 26$ & & 2.78 & Au27-N10 & & 2.16 & $\mathrm{Au} 25-\mathrm{Au} 27$ & & 2.69 \\
\hline $\mathrm{Au} 25-\mathrm{Au} 26$ & & 3.59 & $\mathrm{Au} 26-\mathrm{Au} 27$ & & 2.71 & & & & $\mathrm{Au} 26-\mathrm{Au} 27$ & & 3.18 \\
\hline Au26-Au27 & & 2.99 & & & & & & & & & \\
\hline Au25-Au27 & & 2.69 & & & & & & & & & \\
\hline
\end{tabular}


TABLE S3: Mulliken charges in $\mathrm{xB}(\mathrm{B}=\mathrm{A}, \mathrm{C}, \mathrm{G}, \mathrm{T})$ and $\mathrm{xB}-\mathrm{Au}_{\mathbf{6}}$ complexes at B3LYP/LanL2MB Level.

\begin{tabular}{|c|c|c|c|c|c|c|c|c|c|c|c|}
\hline Atom & $x A$ & $x A-A u_{6}$ & Atom & $x C$ & $x C-A u_{6}$ & Atom & $x G$ & $x G-A u_{6}$ & Atom & $x T$ & $x T-A u_{6}$ \\
\hline $\mathrm{C} 1$ & -0.122 & -0.068 & $\mathrm{C} 1$ & 0.126 & 0.023 & $\mathrm{C} 1$ & -0.175 & -0.077 & $\mathrm{C} 1$ & -0.107 & -0.068 \\
\hline $\mathrm{C} 2$ & 0.199 & 0.108 & $\mathrm{C} 2$ & -0.185 & -0.062 & $\mathrm{C} 2$ & 0.290 & 0.102 & $\mathrm{C} 2$ & 0.325 & 0.111 \\
\hline C3 & 0.116 & -0.011 & $\mathrm{C} 3$ & 0.106 & -0.017 & $\mathrm{C} 3$ & -0.242 & -0.022 & $\mathrm{C} 3$ & 0.019 & -0.020 \\
\hline $\mathrm{C} 4$ & -0.172 & -0.050 & $\mathrm{C} 4$ & 0.317 & 0.117 & $\mathrm{C} 4$ & -0.075 & -0.048 & $\mathrm{C} 4$ & -0.163 & -0.048 \\
\hline $\mathrm{C} 5$ & 0.201 & 0.089 & C5 & -0.101 & -0.064 & $\mathrm{C} 5$ & 0.130 & 0.060 & $\mathrm{C} 5$ & 0.122 & 0.029 \\
\hline C6 & 0.347 & 0.099 & C6 & -0.122 & -0.048 & C6 & 0.352 & 0.095 & C6 & -0.115 & -0.048 \\
\hline N7 & -0.505 & -0.190 & $\mathrm{C} 7$ & 0.496 & 0.246 & $\mathrm{C} 7$ & 0.851 & 0.275 & N7 & -0.664 & -0.257 \\
\hline $\mathrm{C} 8$ & 0.230 & 0.134 & N8 & -0.563 & -0.210 & N8 & -0.837 & -0.256 & $\mathrm{C} 8$ & 0.729 & 0.36 \\
\hline N9 & -0.525 & -0.226 & C9 & 0.666 & 0.344 & C9 & 0.943 & 0.333 & N9 & -0.601 & -0.267 \\
\hline $\mathrm{C} 10$ & 0.478 & 0.215 & N10 & -0.640 & -0.249 & N10 & -0.722 & -0.200 & $\mathrm{C} 10$ & 0.590 & 0.280 \\
\hline N11 & -0.497 & -0.176 & O11 & -0.518 & -0.048 & N11 & -0.557 & -0.21 & ( O11 & -0.495 & -0.129 \\
\hline C12 & 0.299 & 0.159 & N12 & -0.631 & -0.326 & $\mathrm{C} 12$ & 0.366 & 0.104 & $\mathrm{O} 12$ & -0.489 & -0.126 \\
\hline N13 & -0.620 & -0.248 & $\mathrm{C} 13$ & -0.382 & -0.223 & N13 & -0.770 & -0.264 & C13 & -0.382 & -0.221 \\
\hline N14 & -0.639 & -0.323 & H14 & 0.290 & 0.283 & N14 & -0.790 & -0.330 & H14 & 0.290 & 0.273 \\
\hline H15 & 0.119 & 0.160 & H15 & 0.272 & 0.256 & O15 & -0.627 & -0.114 & H15 & 0.275 & 0.289 \\
\hline H16 & 0.281 & 0.261 & H16 & 0.262 & 0.302 & H16 & 0.173 & 0.121 & H16 & 0.116 & 0.130 \\
\hline H17 & 0.273 & 0.269 & H17 & 0.066 & 0.086 & H17 & 0.318 & 0.262 & H17 & 0.089 & 0.110 \\
\hline H18 & 0.091 & 0.140 & H18 & 0.087 & 0.113 & H18 & 0.337 & 0.267 & H18 & 0.126 & 0.10 \\
\hline H19 & 0.084 & 0.141 & H19 & 0.127 & 0.100 & H19 & 0.329 & 0.278 & H19 & 0.125 & 0.10 \\
\hline $\mathrm{H} 20$ & 0.096 & 0.145 & $\mathrm{H} 20$ & 0.127 & 0.100 & $\mathrm{H} 20$ & 0.217 & 0.126 & $\mathrm{H} 20$ & 0.120 & 0.107 \\
\hline $\mathrm{H} 21$ & 0.264 & 0.282 & $\mathrm{H} 21$ & 0.110 & 0.088 & $\mathrm{H} 21$ & 0.166 & 0.123 & $\mathrm{H} 21$ & 0.090 & 0.125 \\
\hline $\mathrm{Au} 22$ & & -0.066 & $\mathrm{H} 22$ & 0.093 & 0.144 & $\mathrm{H} 22$ & 0.321 & 0.253 & $\mathrm{Au} 22$ & & -0.089 \\
\hline $\mathrm{Au} 23$ & & -0.161 & $\mathrm{Au} 23$ & & -0.174 & $\mathrm{Au} 23$ & & -0.224 & $\mathrm{Au} 23$ & & -0.134 \\
\hline $\mathrm{Au} 24$ & & -0.143 & $\mathrm{Au} 24$ & & -0.038 & $\mathrm{Au} 24$ & & -0.187 & $\mathrm{Au} 24$ & & -0.100 \\
\hline $\mathrm{Au} 25$ & & -0.082 & $\mathrm{Au} 25$ & & -0.079 & $\mathrm{Au} 25$ & & -0.015 & $\mathrm{Au} 25$ & & -0.158 \\
\hline $\mathrm{Au} 26$ & & -0.260 & $\mathrm{Au} 26$ & & -0.200 & $\mathrm{Au} 26$ & & -0.214 & $\mathrm{Au} 26$ & & -0.220 \\
\hline \multirow[t]{2}{*}{$\mathrm{Au} 27$} & & -0.195 & $\mathrm{Au} 27$ & & -0.252 & $\mathrm{Au} 27$ & & -0.219 & $\mathrm{Au} 27$ & & -0.123 \\
\hline & & & $\mathrm{Au} 28$ & & -0.209 & $\mathrm{Au} 28$ & & -0.015 & & & \\
\hline
\end{tabular}


TABLE S4: Summary of NBO analysis for $x A$ in gold complexed geometry.

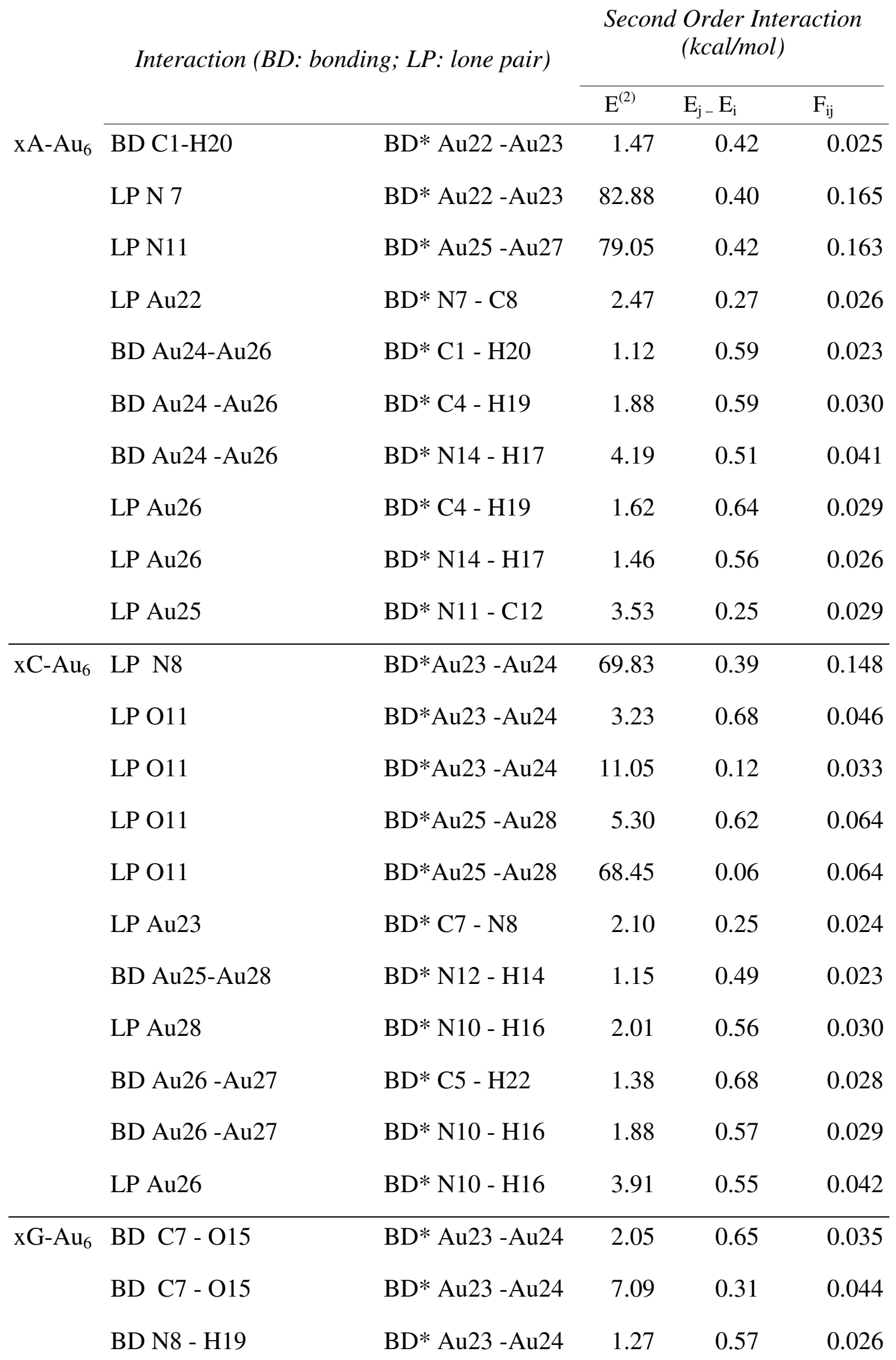



( $\mathrm{kcal} / \mathrm{mol})$

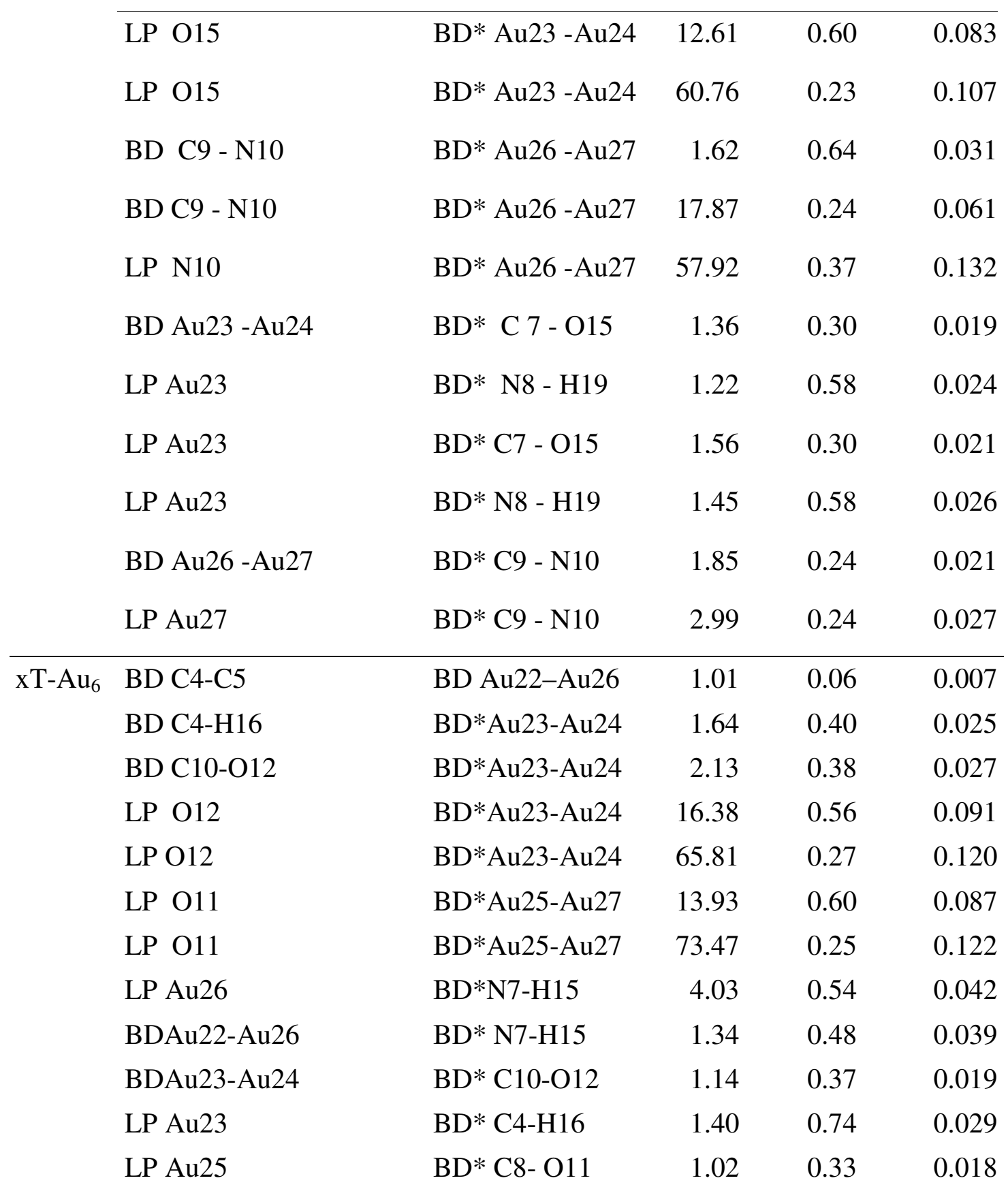


Fig. S1.Optimized Structures of Natural DNA - Bases obtained at B3LYP/LanL2MB Level of Theory (A,C,G, T have the usual meaning)

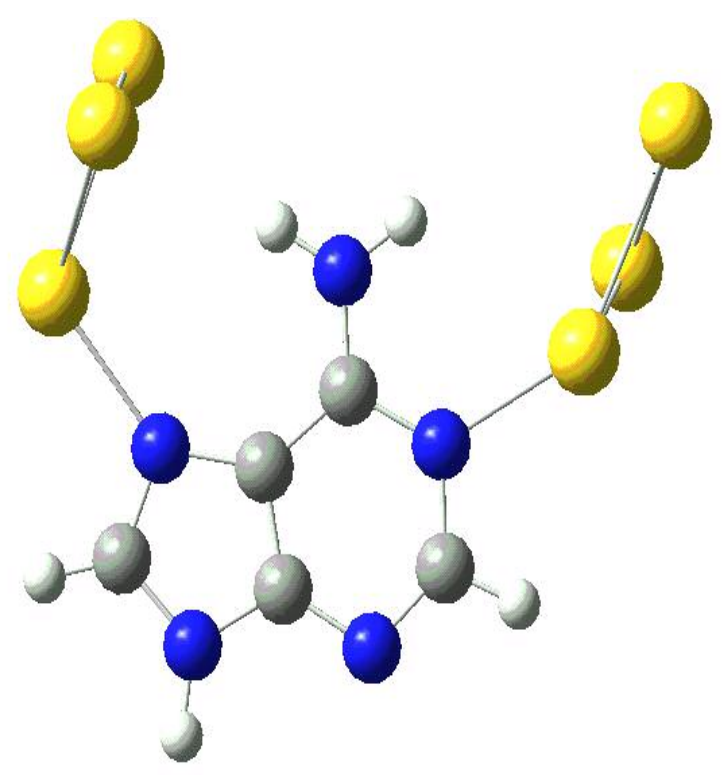

$\mathrm{A}-\mathrm{Au}_{6}$

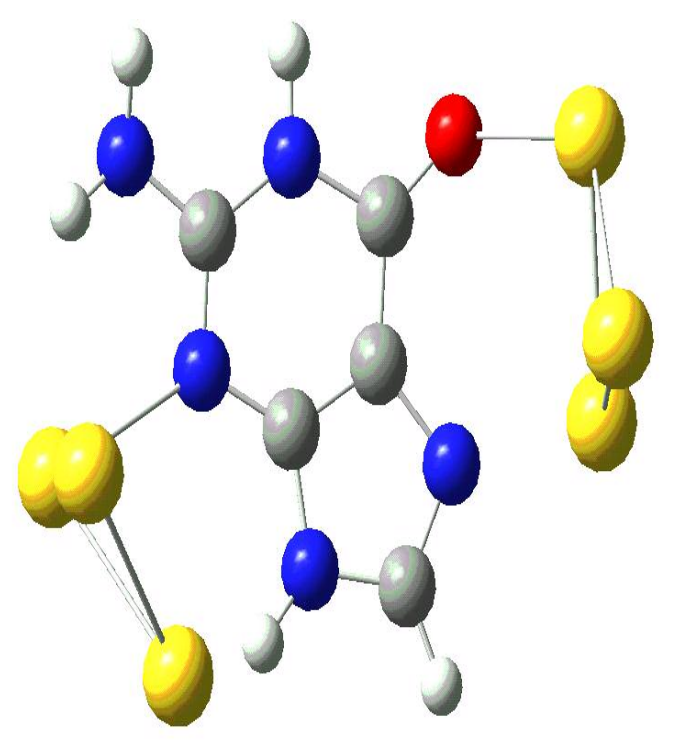

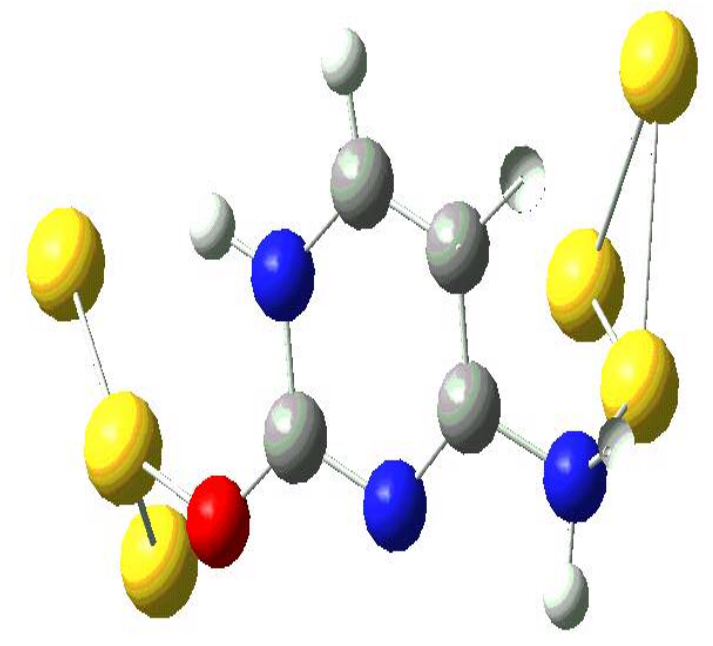

$\mathrm{C}-\mathrm{Au}_{6}$

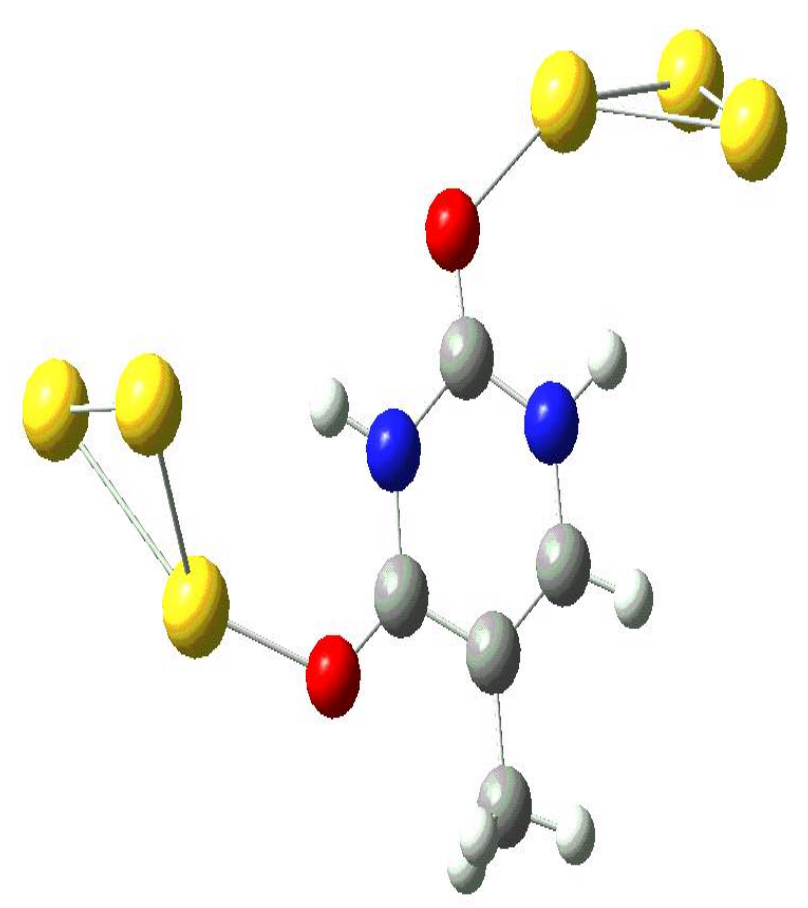

$\mathrm{T}-\mathrm{Au}_{6}$ 